Nao Honda • Norio Miharu • Tetsuaki Hara

Osamu Samura $\cdot$ Hiroshi Honda $\cdot$ Koso Ohama

\title{
Chromosomal FISH analysis of unfertilized human oocytes and polar bodies
}

Received: February 14, 2002 / Accepted: June 10, 2002

\begin{abstract}
The incidence of chromosomal aneuploidy was studied in 208 unfertilized metaphase II human oocytes from an in vitro fertilization program by fluorescence in situ hybridization using probes for chromosomes 18,21 , and X. Chromosome spreads were prepared by a gradual fixationair-drying method. We obtained analyzable results from 183 oocytes and 93 polar bodies; 167 oocytes $(91 \%)$ were normal, 11 (6\%) were diploid, and 5 (3\%) were aneuploid. Of the five aneuploid oocytes, four involved chromosome 21 , and one involved the $\mathrm{X}$ chromosome. In this study, oocyte aneuploidy caused by both nondisjunction of bivalent chromosomes and predivision of univalent chromosomes was observed. The aneuploidy rate $(9.8 \%)$ in the oocytes from women aged $\geqq 35$ years was significantly higher than that $(0.7 \%)$ in those aged 23 to 34 years $(P=$ 0.0017).
\end{abstract}

Key words Oocytes - Chromosome - Aneuploidy · FISH · First polar body $\cdot$ Single chromatids

\section{Introduction}

It has been shown that more than $70 \%$ of human embryos die early in pregnancy (Edmonds et al. 1982), with chromosomal abnormalities being the major cause of the early death. Studies of DNA polymorphisms of trisomic liveborns and abortuses have shown that most human trisomies originate in maternal meiosis (Antonarakis et al. 1992; Fisher et al. 1995; Hassold et al. 1995). For example, the origin of meiotic errors in trisomy 21 is predominantly at the maternal meiosis I division, and that of the errors in trisomy 18 is

N. Honda $(\varangle) \cdot$ N. Miharu $\cdot$ T. Hara $\cdot$ O. Samura $\cdot$ H. Honda

K. Ohama

Department of Obstetrics and Gynecology, Hiroshima University

School of Medicine, 1-2-3 Kasumi, Minami-ku, Hiroshima 734-8551,

Japan

Tel. +81-82-257-5262; Fax: +81-82-257-5264

e-mail: naohonda@hiroshima-u.ac.jp predominantly at the maternal meiosis II division (Hassold et al. 1996; Fisher et al. 1995). A study of 47,XXX females and $47, \mathrm{XXY}$ males of maternal origin demonstrated that $49 \%$ arose during meiosis I, $29 \%$ arose during meiosis II, and $16 \%$ were postzygotic events (Thomas et al. 2001).

It is very important to obtain direct cytogenetic information from oocytes to determine the mechanisms of chromosomal aberrations. Various chromosome studies of human unfertilized oocytes have been reported (Angell 1991; Tarin et al. 1991; Kamiguchi et al. 1993; Nakaoka et al. 1998). In these studies, two mechanisms have been described to explain the occurrence of aneuploidy in the human oocyte, namely, nondisjunction of bivalent chromosomes and predivision of sister chromatids at meiosis I. Recently, through the use of fluorescence in situ hybridization (FISH) technology, cytogenetic information has been obtained from human sperm, embryos, and oocytes. This technique is able to detect chromatids of univalent chromosomes in oocytes and first polar bodies to distinguish between nondisjunction of bivalent chromosomes and predivision of chromatids (Munne et al. 1995; Dailey et al. 1996; Dyban et al. 1996).

In the present study, we performed FISH analysis of unfertilized oocytes and polar bodies with DNA probes specific for chromosomes 18, 21, and $\mathrm{X}$ using a gradual fixation-air-drying method (Kamiguchi et al. 1993), which is a reliable chromosome preparation. The purpose of this study was to determine the incidence of aneuploidy for chromosomes 18,21 , and $\mathrm{X}$ in oocytes, and to know the mechanism of aneuploidy in human oocytes and the relationship between maternal age and the frequency of aneuploidy.

\section{Materials and methods}

Unfertilized oocytes were obtained after informed consent from 84 women who underwent in vitro fertilization (IVF) cycles at three IVF clinics, including one at Hiroshima University. The mean age of the women was 31.7 years, 


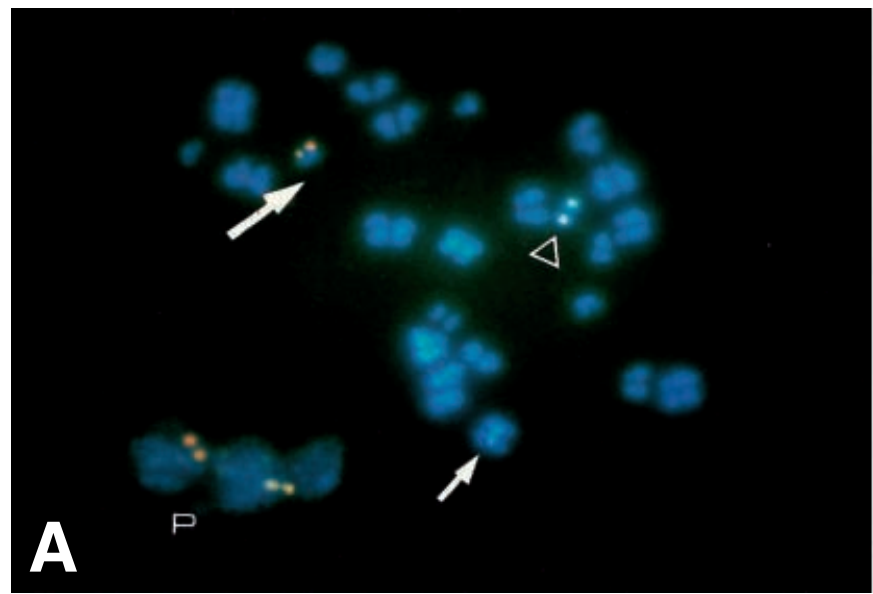

Fig. 1A,B. In situ hybridization of fluorochrome-labelled chromosome 21-, 18-, and X-specific DNA probes to MII oocytes. The probe for the $\mathrm{X}$ chromosome was labelled in green (short arrow), that for chromosome 21 in red (long arrow), and that for chromosome 18 in yellow

ranging from 23 to 45 years. Oocytes were checked for fertilization $18-22 \mathrm{~h}$ after insemination or intracytoplasmic sperm injection. A total of 208 oocytes without pronuclei were considered unfertilized, and therefore included in the study. The oocytes were processed for FISH analysis with a gradual fixation-air-drying method developed by Kamiguchi et al. (1993) to prevent artificial loss of chromosomes during spreads. After removal of the Zona pellucidae with acid tyrode $(\mathrm{pH} 2.0)$, the oocytes were treated with hypotonic solution $(9: 1$ ratio of $0.9 \%$ sodium citrate $: 30 \%$ fetal bovine serum) at $37^{\circ} \mathrm{C}$ for $20 \mathrm{~min}$. The oocytes were transferred to fixative $(5: 1: 4$ ratio of methanol:acetic acid:distilled water) for 3-5 min. The oocytes were then placed gently onto a clean slide and covered with a flow of fixative (3:1 ratio of methanol:acetic acid). The slides were placed in a Coplin jar filled with the fixative, transferred to another Coplin jar with a 3:3:1 ratio of methanol:acetic acid: distilled water fixative for $1 \mathrm{~min}$, and dried with warm air.

Directly fluorescence-labeled commercial probes specific for chromosome 18 (CEP $18 \alpha$-satellite, Spectrum Green and Orange, Vysis, Downers Grove, IL, USA), chromosome 21 (LSI 21, DNA sequences complementary to the 21q22.13-21q22.2 region, Spectrum Orange, Vysis), and Xchromosome (CEP X $\alpha$-satellite, Spectrum Green, Vysis) were used. The slides and the probes were denatured separately in $70 \%$ formamide $/ 2 \times \mathrm{SSC}$ at $75^{\circ} \mathrm{C}$ for 3 to $5 \mathrm{~min}$. Probe cocktail $(3 \mu \mathrm{l})$ was added to the slides and sealed under $9-\mathrm{mm}^{2}$ coverslips. After overnight incubation at $37^{\circ} \mathrm{C}$ in a humidified chamber, the slides were washed twice in $2 \times \mathrm{SSC}$ and $50 \%$ formamide at $40^{\circ} \mathrm{C}$ for $3 \mathrm{~min}$. Chromosomes were counterstained with 4'6-diamidino-2phenylindole (DAPI, Sigma, St. Louis, MO, USA). Hybridization signals in oocyte chromosomes and polar bodies were visualized under a Nikon Axiophot microscope (Nikon, Tokyo, Japan) fitted with a triple-band pass filter set (Chroma Technology, Bratteboro, VT, USA).

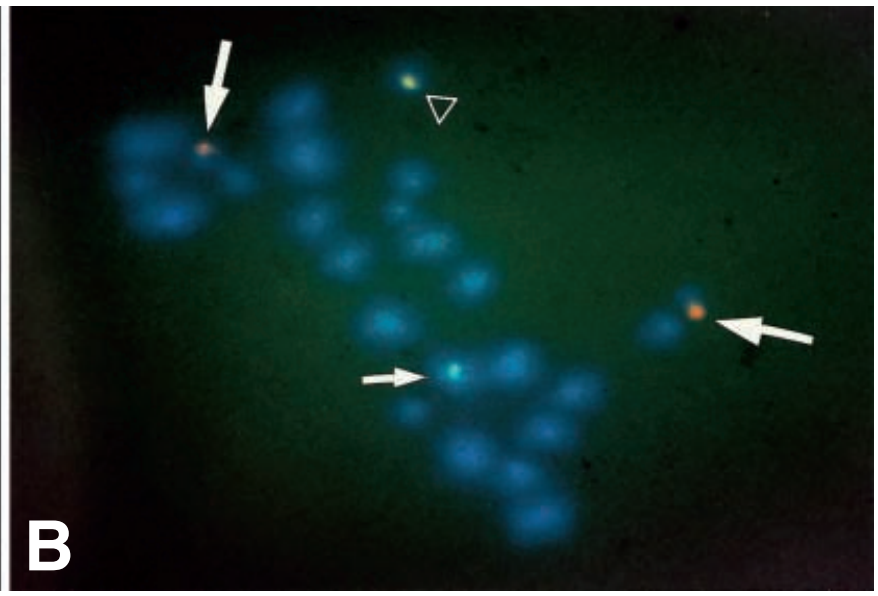

(triangle). A Example of fluorescence in situ hybridization analysis on unfertilized oocytes and first polar body $(P)$. A normal haploid metaphase hybridized with chromosomes 21,18 , and X. B Two chromosome 21 univalents (with two chromatids each) were found

Each oocyte and polar body chromosome consists of two chromatids, and each chromatid gives rise to one fluorescent signal. Probes to chromosomes 18 and $\mathrm{X}$ are complementary to centromeric regions, and therefore give two paired signals or two distinct signals if chromatids are separating. The probe to chromosome 21 is complementary to regions on the long arms of the chromosome, and will always show some degree of signal separation.

\section{Results}

Of the 208 unfertilized oocytes studied, 183 showed detectable FISH signals, representing chromosomes 18, 21, and X (Fig. 1A). The mean age of the 71 women whose oocytes showed analyzable signals was 31.4 years with a range of 2345 years. The other 25 oocytes could not be analyzed because of degraded chromosomal material or overlapping signals. Of the 183 oocytes analyzed, 167 (91.2\%) were normal, $11(6.0 \%)$ were diploid, and $5(2.7 \%)$ were aneuploid. First polar bodies were also analyzed in 93 of these oocytes, 87 of which showed an expected number of FISH signals. Of the remaining six oocytes, two were aneuploid and four were diploid. In two of the four diploid oocytes, the corresponding polar bodies were diploid (four signals for two of the 18,21, and X chromosomes), and in the remaining two diploid oocytes, the corresponding polar bodies were haploid (two signals each for chromosomes 18, 21 , and X). Of the five oocytes that were found to be aneuploid, two had polar bodies with informative FISH signals (Table 1). In two aneuploid oocytes, discordance was revealed between signals in the oocyte and the polar body. One oocyte showed signals for two chromosome 21s, and the polar body had none, indicating the occurrence of first meiotic nondisjunction (Fig. 1B). The other oocyte had a single chromatid for chromosome 21, whereas the polar 
Table 1. Women showing abnormal FISH signals in oocytes and their first polar bodies

\begin{tabular}{rlll}
\hline Women & $\begin{array}{l}\text { Maternal } \\
\text { age } \\
\text { (years) }\end{array}$ & $\begin{array}{l}\text { Chromosomal } \\
\text { abnormalities in oocyte }\end{array}$ & Polar body \\
\hline 1 & 45 & Extra 21 univalent & No chromosome 21 \\
2 & 38 & Missing a chromatid 21 & Extra chromatid 21 \\
3 & 38 & Missing 21 univalent & NA \\
4 & 41 & Missing a chromatid 21 & NA \\
5 & 25 & Missing an X chromatid & NA \\
6 & 33 & Diploid & Haploid \\
7 & 36 & Diploid & Haploid \\
8 & 23 & Diploid & Diploid \\
9 & 30 & Diploid & Diploid \\
10 & 29 & Diploid & NA \\
11 & 30 & Diploid & NA \\
12 & 30 & Diploid & NA \\
13 & 32 & Diploid & NA \\
14 & 32 & Diploid & NA \\
15 & 34 & Diploid & NA \\
16 & 42 & Diploid & NA \\
\hline
\end{tabular}

FISH, Fluorescence in situ hybridization; NA, not analyzed

${ }^{a}$ Haploid in polar body, two signals in each chromosome; diploid in polar body, four signals in each chromosome

body showed three signals corresponding to a chromosome plus a chromatid 21 (predivision at first meiosis). All of the oocytes with chromosome 21 aneuploidy were from women aged 38-45 years. An oocyte from a 25 -year-old woman showed a single $\mathrm{X}$ chromosome signal caused by a chromatid, suggesting the presence of predivision of the X chromosome. The polar body of the oocyte was not analyzable. Oocytes with aneuploidy for chromosome 18 were not encountered. Precocious separation of sister chromatids was observed in several oocytes, but was considered to be due to chromosomal degradation during culturing.

The oocytes were classified into two groups according maternal age : $23-34$ years $(n=142)$, and $\geqq 35$ years of age $(n=41)$. The aneuploidy rate, including both nondisjunction and predivision, was significantly higher in oocytes from the $\geqq 35$ years' group as compared with the younger age group $(P=0.0017)$ (Table 2$)$. However, significant differences were not observed in the incidence of either nondisjunction or predivision between the two groups.

\section{Discussion}

In the present study, we used a reliable method, a gradual fixation-air-drying method (Kamiguchi et al. 1993), and investigated the incidence of chromosomal abnormalities in unfertilized oocytes using FISH. To our knowledge, this work represents the analysis of the largest number of unfertilized human oocytes by FISH using the gradual fixationair-drying method to date. Most previous studies of human oocytes by FISH have been performed according to Tarkowski's fixation method (Tarkowski 1966), in which artificial loss of chromosomes might occur during the process of slide preparation (Munne et al. 1995; Dailey et al. 1996; Dyban et al. 1996; Martini et al. 1997; Benzacken et al.
Table 2 Chromosome abnormality rate and maternal age

\begin{tabular}{lcl}
\hline & \multicolumn{2}{l}{ Maternal age (years) } \\
\cline { 2 - 3 } Chromosome & $\begin{array}{ll}23-34 \\
(n=142)\end{array}$ & $\begin{array}{l}\geqq 35 \\
(n=41)\end{array}$ \\
\hline Normal & $132(93.0 \%)$ & $35(85.4 \%)$ \\
Abnormal & $10(7.0 \%)$ & $6(14.6 \%)$ \\
Aneuploidy & $1(0.7 \%)^{\mathrm{a}}$ & $4(9.8 \%)^{\mathrm{a}}$ \\
Diploidy & $9(6.3 \%)$ & $2(4.8 \%)$ \\
\hline
\end{tabular}

${ }^{a}$ Aneuploidy rate in the group of older women was significantly higher than that in younger women $(P<0.05)$

1998; Mahmood et al. 2000). Polar bodies were also analyzed to reveal nondisjunction or predivision at meiosis I, because independent segregation of chromosomes in oocytes and the corresponding polar bodies should yield an equal incidence of hypohaploid and hyperhaploid oocytes. Because chromosomes are difficult to recognize clearly, FISH analysis was very useful for determining chromosome aneuploidy, especially in polar bodies.

In our study, the incidence of aneuploid oocytes for chromosomes 18,21 , and $X$ in women aged $\geqq 35$ years was $9.8 \%$, which was lower than that observed in previous reports (18.8\% for chromosomes X, 18, and 13/21 by Dailey et al. 1996; $19.0 \%$ for chromosomes 21 and X by Benzaken et al. 1998). This discrepancy may be due to the methods used for chromosome preparation (i.e., the gradual fixation-airdrying method vs. Tarkowski's method) or to differences in the sample size. For chromosome 21, the incidence of aneuploid oocytes in the present study $(2.1 \%)$ is comparable with our previous study, which was done by karyotyping, assuming that a half of group $\mathrm{G}$ aneuploidies were derived from chromosome 21 (i.e., 1.6\%). We did not find any oocytes with chromosome 18 aneuploidy, a finding that might be due to the small sample size or to the relatively low incidence of nondisjunction of maternal meiosis I involving chromosome 18. A previous examination of trisomic fetuses and liveborn individuals by the analysis of DNA polymorphisms showed that, in cases of trisomy 21 , nondisjunction in maternal meiosis I and meiosis II was responsible for $65 \%$ and $23 \%$ of this aneuploidy, respectively (Hassold et al. 1996). Conversely, with trisomy $18,33 \%$ were attributed to an error at maternal meiosis I, $56 \%$ to an error at meiosis II, and $11 \%$ to mitotic errors (Hassold et al. 1996).

We observed two diploid oocytes with haploid polar bodies. There are two mechanisms that may have produced these diploid oocytes: (1) A tetraploid oocyte produced by cytoplasmic fusion of two oogonia underwent normal meiotic division, and one of two polar bodies was lost during culturing or removal of the zona pellucida; (2) A diploid polar body was separated by degeneration and lost during culturing or fixation. Seven diploid oocytes had no polar bodies, most likely resulting from nondivision (failure of exclusion of the first polar body) at first meiosis.

In our study, both absent univalents because of nondisjunction and single chromatids after predivision were found in aneuploid oocytes, with both nondisjunction and predivision being confirmed by the results of FISH analysis in polar bodies. Nondisjunction at meiosis I results in the 
gain or loss of a bivalent. Premature division of a bivalent at meiosis I may be followed by the gain or loss of a univalent at meiosis II in 50\% of instances. Angell (1991) and Angell et al. (1994) suggested that predivision is the only source of aneuploidy in human oocytes. However, in other previous studies, oocyte aneuploidy as a result of both the nondisjunction of bivalent chromosomes and the predivision of univalent chromosomes was observed (Kamiguchi et al. 1993; Lim et al. 1995; Daily et al. 1996; Nakaoka et al. 1998).

The present study indicated that aneuploidy for chromosome 21 at meiosis II is maternal-age dependent, because four oocytes showing chromosome 21 aneuploidy were observed in the older group, whereas none were observed in the younger group. Benzacken et al. (1998) reported that the aneuploidy rate of chromosome 21 increased significantly from $8.1 \%$ in 25 - to 30 -year-old women to $11.9 \%$, $17.6 \%$, and $25 \%$, respectively, in $31-$ to $35-$, $36-$ to $40-$, and $>40$-year-old women, respectively. Therefore, these researchers suggested a correlation between maternal age and the aneuploidy rate of chromosome 21 .

Other studies of unfertilized oocytes, on the other hand, failed to confirm this relationship (Kamiguchi et al. 1993; Angell et al. 1993; Almeida et al. 1994; Nakaoka et al. 1998). This may be because our study analyzed for chromosomes 18,21 , and $\mathrm{X}$ by FISH, whereas other studies analyzed each chromosome group. Moreover, the sample size was different for each age group.

We found no significant relation to maternal age for nondisjunction and predivision, which might be due to the small sample size used. Dailey et al. (1996), using FISH analysis of chromosomes $13 / 21,18$, and X, reported that the frequencies of nondisjunction of bivalents increased with increasing maternal age, whereas unbalanced predivision of single chromatids was independent of maternal age. Because no one but Dailey et al. (1996) has shown a maternal age effect on aneuploidy discriminating between nondisjunction and predivision to date, further analyses of large numbers of oocytes will be expected to study the maternal age effect on nondisjunction and predivision.

In conclusion, our study confirms the high incidence of chromosome 21 abnormalities, probably related to maternal age. However, chromosome 18 abnormalities were not detected, thereby suggesting that nondisjunction of chromosome 18 may occur predominantly during meiosis II. More data are needed to confirm the effect of maternal age on the frequency of chromosome 21 abnormalities, and additional FISH analyses should be performed to identify the chromosomes involved in the aneuploidies.

Acknowledgments We wish to thank Dr. Yujiro Kamiguchi at Asahikawa Medical College, Department of Biological Science, Japan for his many helpful comments, Dr. Masaaki Takenaka and Dr. Katsuhiko Takahashi for providing unfertilized human oocytes, and Dr. Kirby Johnson for reviewing this manuscript. This study was supported in part by Grants-in-aid for Scientific Research from the Ministry of Education, Science, Sports and Culture of Japan (No.09671685) and by JAOG Ogyaa Donation Foundation (JODF;1995).

\section{References}

Almeida PA, Bolton VN (1994) The relationship between chromosomal abnormalities in the human oocyte and fertilization in vitro. Hum Reprod 9:343-346

Angell RR (1991) Predivision in human oocytes at meiosis I: a mechanism for trisomy formation in man. Hum Genet 86:383-387

Angell RR, Xian J, Keith J (1993) Chromosome anomalities in human oocytes in relation to age. Hum Reprod 8:1047-1054

Angell RR, Xian J, Keith J, Ledger W, Baird DT (1994) First meiotic division abnormalities in human oocytes: mechanism of trisomy formation. Cytogenet Cell Genet 65:194-202

Antonarakis S, Peterson MB, Mclnnis MG, Adelsberger PA, Schinzel AA, Binkert F, Pangalos C, Raoul O, Slaugenhaupt SA, Hafez M, Cohen MM, Roulson D, Schwartz S, Mikkelsen M, Tranebjaerg L, Greenberg F, Hoar DI, Rudd NL, Warren AC, Metaxotou C, Bartsocas C, Chakravarti A (1992) The meiotic stage of nondisjunction in trisomy 21: determination by using DNA polymorphisms. Am J Hum Genet 50:544-550

Benzacken B, Martin-Pont B, Bergere M, Hugues JN, Wolf JP, Selva J (1998) Chromosome 21 detection in human oocyte fluorescence in situ hybridization: possible effect of maternal age. J Assist Reprod Genet 15:105-110

Dailey T, Dale B, Cohen J, Munne S (1996) Association between nondisjunction and maternal age in meiosis I human oocytes. Am J Hum Genet 59:176-184

Dyban A, Freidine M, Severova E, Cieslak J, Ivakhnenko V, Verlinsky Y (1996) Detection of aneuploidy in human oocytes and corresponding first polar bodies by fluorescent in situ hybridization. J Assist Reprod Genet 13:73-78

Edmonds DK, Lindsay KS, Miller JF, Williamson E, Wood PJ (1982) Early embryonic mortality in women. Fertil Steril 38:447-453

Fisher JM, Harvey JF, Morton NE, Jacobs PA (1995) Trisomy 18: studies of the parent and cell division of origin and the effect of aberrant recombination on nondisjunction. Am J Hum Genet 56:669-675

Hassold T, Merrill M, Adkins K, Freeman S, Sherman S (1995) Recombination and maternal age-dependent nondisjunction: molecular studies of trisomy 16. Am J Hum Genet 57:867-874

Hassold T, Abruzzo M, Adkins K, Griffin D, Merrill M, Millie E, Saker D, Shen J, Zaragoza M (1996) Human aneuploidy: incidence, origin, and etiology. Environ Mol Mutagen 28:167-175

Kamiguchi Y, Rosenbusch B, Sterzik K, Mikamo K (1993) Chromosome analysis of unfertilized human oocytes prepared by a gradual fixation-air drying method. Hum Genet 90:533-541

Lim AS, Ho AT, Tsakok MF (1995) Chromosomes of oocytes failing in-vitro fertilization. Hum Reprod 10:2570-2575

Mahmood R, Brierley CH, Faed MJ, Mills JA, Delhanty JD (2000) Mechanisms of maternal aneuploidy: FISH analysis of oocytes and polar bodies in patients undergoing assisted conception. Hum Genet 106:620-626

Martini E, Flaherty SP, Swann NJ, Payne D, Matthews CD (1997) Analysis of unfertilized oocytes subjected to intracytoplasmic sperm injection using two rounds of fluorescence in-situ hybridization and probes to five chromosomes. Hum Reprod 12:2011-2018

Munne S, Dailey T, Sultan KM, Grifo J, Cohen J (1995) The use of first polar bodies for preimplantation diagnosis of aneuploidy. Hum Reprod 10:1014-1020

Nakaoka Y, Okamoto E, Miharu N, Ohama K (1998) Chromosome analysis in human oocytes remaining unfertilized after in-vitro insemination: effect of maternal age and fertilization rate. Hum Reprod 13:419-424

Tarin JJ, Gomez E, Sampaio M, Ruiz M, Remohi J, Pellicer A (1991) Cytogenetic analysis of human oocytes from fertile women. Hum Reprod 6:1100-1103

Tarkowski AK (1966) An air-drying method for chromosome preparation from mouse eggs. Cytogenetics 5:394-400

Thomas NS, Ennis S, Sharp AJ, Durkie M, Hassold TJ, Collins AR, Jacobs PA (2001) Maternal sex chromosome nondisjunction: evidence for X chromosome-specific risk factors. Hum Mol Genet 10:243-250 